Lawrence M. Gibbs, MD, MSEd; Rajasree J. Nair, MD

Methodist Charlton Family Medicine Residency, Dallas, TX

Joan Nashelsky, MLS

Family Physicians Inquiries Network, lowa City

ASSISTANT EDITOR Rick Guthmann, MD, MPH

Advocate Illinois Masonic Family Medicine Residency, Chicago

\title{
Q/ Does evidence support the use of supplements to aid in $\mathrm{BP}$ control?
}

\section{EVIDENCE-BASED ANSWER}

A YES. A number of well-tolerated natural therapies have been shown to reduce systolic and diastolic blood pressure (BP). (See TABLE ${ }^{1-8}$ for summary.) However, the studies don't provide direct evidence of whether the decrease in BP is

\section{Evidence summary}

- Cocoa. A 2017 Cochrane review evaluated data from more than 1800 patients (401 in hypertension studies) to determine the effect of cocoa on BP. ${ }^{1}$ Compared with placebo (in flavanol-free or low-flavanol controls), cocoa lowered systolic BP by $1.8 \mathrm{~mm} \mathrm{Hg}$ (confidence interval $[\mathrm{CI}],-3.1$ to -0.4$)$ and diastolic BP by $1.8 \mathrm{~mm} \mathrm{Hg}(\mathrm{CI},-2.6$ to -0.9$)$. Further analysis of patients with hypertension (only) showed a reduction in systolic BP of $4 \mathrm{~mm} \mathrm{Hg}$ (CI, -6.7 to -1.3 ).

Omega-3 fatty acids. Similarly, a 2014 meta-analysis investigating omega-3 fatty acids (eicosapentaenoic acid [EPA] + docosahexaenoic acid [DHA]) included data from 4489 patients (956 with hypertension) and showed reductions in systolic BP of $1.5 \mathrm{~mm} \mathrm{Hg}$ (CI, -2.3 to -0.8 ) and diastolic BP of $1 \mathrm{~mm} \mathrm{Hg}$ (CI, -1.5 to -0.4$)$, compared with placebo. ${ }^{2}$ Again, subgroup analysis of patients with hypertension (only) at baseline revealed a greater decrease in systolic and diastolic BP: $4.5 \mathrm{~mm} \mathrm{Hg}$ (CI, -6.1 to -2.8 ) and $3.1 \mathrm{~mm} \mathrm{Hg}$ (CI, -4.4 to -1.8 ), respectively.

Garlic and potassium chloride. Separate meta-analyses that included only patients with hypertension found that both garlic and potassium significantly lowered $\mathrm{BP}^{3,4}$ A 2015 meta-analysis comparing a vari- linked to patient-oriented outcomes. Nor do they allow definitive conclusions concerning the lasting nature of the reductions, because most studies were fewer than 6 months in duration (strength of recommendation: $\mathbf{C}$, disease-oriented evidence).

ety of garlic preparations with placebo in patients with hypertension showed decreases in systolic BP of $9.1 \mathrm{~mm} \mathrm{Hg}$ (CI, -12.7 to -5.4) and in diastolic BP of $3.8 \mathrm{~mm} \mathrm{Hg}$ (CI, -6.7 to -1$){ }^{3}$ Meanwhile, a meta-analysis in 2017 comparing different doses of potassium chloride with placebo demonstrated reductions in systolic BP of $4.3 \mathrm{~mm} \mathrm{Hg}(\mathrm{CI},-6$ to -2.5$)$ and diastolic BP of $2.5 \mathrm{~mm} \mathrm{Hg}$ (CI, -4.1 to -1$){ }^{4}$

L-arginine. Another meta-analysis of randomized controlled trials reported evidence that oral L-arginine, compared with placebo, significantly reduced systolic BP by $5.4 \mathrm{~mm} \mathrm{Hg}$ (CI, -8.5 to -2.3 ) and diastolic $\mathrm{BP}$ by $2.7 \mathrm{~mm} \mathrm{Hg}$ (CI, -3.8 to -1.5$).{ }^{5}$ Close to one-third of patients had hypertension at baseline.

I Beetroot juice. A double-blind, placebocontrolled study showed that consumption of beetroot juice (with nitrate) once daily reduced BP in 3 different settings (clinic, 24-hour ambulatory, and home readings) when compared with placebo (nitrate-free beetroot juice). ${ }^{6}$ Study participants were mostly British women, overweight, without significant cardiovascular or renal disease, and with uncontrolled ambulatory BP (> 135/85 mm Hg).

I Flax seed. A prospective, double-blind trial of patients with peripheral artery disease compared the antihypertensive effects 
TABLE

\section{How well do these supplements aid in BP control?}

\begin{tabular}{|c|c|c|c|c|c|c|}
\hline $\begin{array}{l}\text { Therapy } \\
\text { (study type) }\end{array}$ & Subjects & Dose & $\begin{array}{l}\text { Duration } \\
\text { (wk) }\end{array}$ & Adverse effects & $\begin{array}{l}\text { Effect on } \mathrm{BP} \text {, reduction } \\
\text { in } \mathrm{mm} \mathrm{Hg}\end{array}$ & Comments \\
\hline $\begin{array}{l}\text { Beetroot/ } \\
\text { nitrate }^{6} \\
(\mathrm{RCT})\end{array}$ & $\begin{array}{l}68 \\
\text { (all with HTN; } \\
\text { half drug } \\
\text { naïve and half } \\
\text { medication } \\
\text { treated) }\end{array}$ & $\begin{array}{l}\text { Beetroot } \\
\text { juice } 250 \\
\mathrm{~mL} / \mathrm{d} \\
(\sim 6.4 \mathrm{mmoL} \\
\text { nitrate })\end{array}$ & 4 & $\begin{array}{l}\text { Beeturia and fecal } \\
\text { discoloration } \\
\text { (mild) }\end{array}$ & $\begin{array}{l}\text { Mean: } \\
\text { SBP }=7.7(\mathrm{Cl},-11.8 \text { to }-3.6 ; \\
P<.001) \\
\mathrm{DBP}=2.4(\mathrm{Cl},-4.9 \text { to } 0.0 ; \\
P=.050) \\
24-\mathrm{hr} \mathrm{BP}: \\
\mathrm{SBP}=7.7(\mathrm{Cl},-11.2 \text { to }-4.1 ; \\
P<.001) \\
\mathrm{DBP}=5.2(\mathrm{Cl},-7.7-2.7 ; \\
P<0.001) \\
\mathrm{Home} \mathrm{BP}: \\
\mathrm{SBP}=8.1(\mathrm{Cl},-12.4 \text { to }-3.8 \\
P<.001) \\
\mathrm{DBP}=3.8(\mathrm{Cl},-6.9 \text { to }-0.7 \\
P<.01)\end{array}$ & $\begin{array}{l}\text { No nitrate- } \\
\text { associated } \\
\text { tachyphylaxis was } \\
\text { noted }\end{array}$ \\
\hline $\begin{array}{l}\text { Cocoa }^{1} \\
\text { (SR/MA) }\end{array}$ & $\begin{array}{l}1804 \\
\text { (401 with } \\
\text { HTN) }\end{array}$ & $\begin{array}{l}1.4-105 \mathrm{~g} / \mathrm{d} \\
\text { of cocoa } \\
\text { products }\end{array}$ & $\begin{array}{l}\text { 2-18 } \\
\text { (mean, 9) }\end{array}$ & $\begin{array}{l}\text { Mild GI symptoms } \\
\text { including nausea }\end{array}$ & $\begin{array}{l}\mathrm{SBP}=1.8(\mathrm{Cl},-3.1 \text { to }-0.4) \\
\mathrm{DBP}=1.8(\mathrm{Cl},-2.6 \text { to }-0.9) \\
\text { HTN subgroup: } \\
\mathrm{SBP}=4(\mathrm{Cl},-6.7 \text { to }-1.3) \\
\text { DBP N/A }\end{array}$ & $\begin{array}{l}\text { Moderate- } \\
\text { quality evidence; } \\
\text { moderate-high } \\
\text { heterogeneity; mild } \\
\text { reporting bias }\end{array}$ \\
\hline $\begin{array}{l}\text { Flax seed }^{7} \\
\text { (prospective } \\
\text { RCT) }\end{array}$ & $\begin{array}{l}110 \\
\text { (all with HTN) }\end{array}$ & $30 \mathrm{~g} / \mathrm{d}$ & 24 & Similar to placebo & $\begin{array}{l}\mathrm{SBP}=10(P=.04) \\
\mathrm{DBP}=7(P=.004)\end{array}$ & $\begin{array}{l}\text { All patients had } \\
\text { PAD; BP reductions } \\
\text { were more } \\
\text { pronounced in } \\
\text { patients with HTN }\end{array}$ \\
\hline $\begin{array}{l}\text { Garlic }^{3} \\
\text { (SR/MA, } \\
9 \text { RCTs) }\end{array}$ & $\begin{array}{l}482 \\
\text { (all with HTN) }\end{array}$ & $\begin{array}{l}240-2400 \\
\text { mg/d } \\
\text { (aged } \\
\text { extract, garlic } \\
\text { oil, garlic } \\
\text { powder, egg } \\
\text { yolk-enriched } \\
\text { garlic } \\
\text { powder) }\end{array}$ & $\begin{array}{l}\text { 8-26 } \\
\text { (mean, } \\
13.5 \text { ) }\end{array}$ & $\begin{array}{l}\text { Mild GI symptoms } \\
\text { such as burping, } \\
\text { flatulence, and } \\
\text { reflux }\end{array}$ & $\begin{array}{l}\mathrm{SBP}=9.1(\mathrm{Cl},-12.7 \text { to }-5.4 ; \\
P=.0006) \\
\mathrm{DBP}=3.8(\mathrm{Cl},-6.7 \text { to }-1 ; \\
P=.00001)\end{array}$ & $\begin{array}{l}\text { Quality of } \\
\text { included trials } \\
\text { was moderate; } \\
\text { moderate-high } \\
\text { heterogeneity; } \\
\text { insufficient data } \\
\text { to determine } \\
\text { publication bias }\end{array}$ \\
\hline $\begin{array}{l}\text { L-arginine } \\
\text { (SR/MA, } \\
11 \text { RCTs) }\end{array}$ & $\begin{array}{l}387 \\
(1 / 3 \text { with } \\
\text { baseline HTN) }\end{array}$ & $\begin{array}{l}\text { 4-24 g/d } \\
\text { (mean, } 9 \mathrm{~g} / \mathrm{d})\end{array}$ & $\begin{array}{l}2-24 \\
\text { (mean, 4) }\end{array}$ & Diarrhea & $\begin{array}{l}\mathrm{SBP}=5.4(\mathrm{Cl},-8.5 \text { to }-2.3 \\
P=.001) \\
\mathrm{DBP}=2.7(\mathrm{Cl},-3.8 \text { to }-1.5 ; \\
P<.001)\end{array}$ & $\begin{array}{l}\text { All studies were } \\
\text { double-blind; BP } \\
\text { primary outcome in } \\
5 \text { studies; moderate } \\
\text { heterogeneity; } \\
\text { greater BP } \\
\text { reduction in } \\
\text { patients with } \\
\text { higher baseline BP }\end{array}$ \\
\hline $\begin{array}{l}\text { Olive leaf8 } \\
(R C T)\end{array}$ & $\begin{array}{l}148 \\
\text { (all with HTN) }\end{array}$ & $\begin{array}{l}\text { Olive leaf } \\
\text { extract } \\
500 \mathrm{mg} \text { bid; } \\
\text { captopril } \\
12.5-25 \mathrm{mg}\end{array}$ & 8 & $\begin{array}{l}\text { Cough, vertigo, } \\
\text { myalgias, and } \\
\text { headache (mild } \\
\text { and similar } \\
\text { between groups) }\end{array}$ & $\begin{array}{l}\text { Olive leaf extract: } \\
\mathrm{SBP}=11.5 \pm 8.6 \\
\mathrm{DBP}=4.8 \pm 5.5 \\
\text { Captopril: } \\
\mathrm{SBP}=13.7 \pm 7.6 \\
\mathrm{DBP}=6.4 \pm 5.2 \text { (Difference } \\
\text { between treatments } \\
P>.05 \text { ) }\end{array}$ & $\begin{array}{l}\text { Study excluded } \\
\text { patients with signs } \\
\text { of target organ } \\
\text { damage (eg, heart, } \\
\text { kidneys, liver) }\end{array}$ \\
\hline
\end{tabular}


TABLE

\section{How well do these supplements aid in BP control? (cont'd)}

\begin{tabular}{|c|c|c|c|c|c|c|}
\hline $\begin{array}{l}\text { Therapy } \\
\text { (study type) }\end{array}$ & Subjects & Dose & $\begin{array}{l}\text { Duration } \\
\text { (wk) }\end{array}$ & Adverse effects & $\begin{array}{l}\text { Effect on BP, reduction } \\
\text { in } \mathrm{mm} \mathrm{Hg}\end{array}$ & Comments \\
\hline $\begin{array}{l}\text { Omega-3 } \\
\text { fatty acids } \\
\text { (EPA+DHA) } \\
\text { (SR/MA, } \\
70 \text { RCTs) }\end{array}$ & $\begin{array}{l}4489 \\
\text { (956 with } \\
\text { HTN) }\end{array}$ & $\begin{array}{l}\text { EPA+DHA } \\
\text { dose: } 0.1-15 \\
\text { g/d (mean, } \\
3.8 \mathrm{~g} / \mathrm{d} \text { ) }\end{array}$ & $\begin{array}{l}4-52 \\
\text { (mean, } \\
10)\end{array}$ & NR & $\begin{array}{l}\text { Overall: } \\
\text { SBP }=1.5(\mathrm{Cl},-2.3 \text { to }-0.8) \\
\text { DBP }=1(\mathrm{Cl},-1.5 \text { to }-0.4) \\
\text { HTN subjects: } \\
\text { SBP }=4.5(\mathrm{Cl},-6.1 \text { to }-2.8) \\
\text { DBP }=3.1(\mathrm{Cl},-4.4 \text { to }-1.8)\end{array}$ & $\begin{array}{l}\text { All trials were } \\
\text { double-blind; low } \\
\text { heterogeneity; mild } \\
\text { publication bias }\end{array}$ \\
\hline $\begin{array}{l}\text { Potassium } \\
\text { (SR/MA, } \\
23 \text { RCTs) }\end{array}$ & $\begin{array}{l}1213 \\
\text { (all with HTN) }\end{array}$ & $\begin{array}{l}6-200 \mathrm{mEq} \text { of } \\
\mathrm{KCl} \text { daily }\end{array}$ & $4-52$ & $\begin{array}{l}\text { Abdominal pain, } \\
\text { nausea, vomiting, } \\
\text { diarrhea, gas. } \\
\text { (mild, similar to } \\
\text { placebo) }\end{array}$ & $\begin{array}{l}\mathrm{SBP}=4.3(\mathrm{Cl},-6.0 \text { to }-2.5 ; \\
P<.00001) \\
\mathrm{DBP}=2.5(\mathrm{Cl},-4.1 \text { to }-1.0 ; \\
P<.001) \\
\text { Mean change data: } \\
\mathrm{SBP}=8.9(\mathrm{Cl},-13.7 \text { to }-4.1 ; \\
P=.0003) ; \\
\mathrm{DBP}=6.4(\mathrm{Cl},-11 \text { to }-1.8 ; \\
P=.006)\end{array}$ & $\begin{array}{l}\text { Mild-moderate } \\
\text { heterogeneity; } \\
\text { low bias; showed } \\
\text { dose-response } \\
\text { relationship }\end{array}$ \\
\hline
\end{tabular}

BP, blood pressure; $\mathrm{Cl}, 95 \%$ confidence interval; DBP, diastolic blood pressure; EPA+DHA, eicosapentaenoic acid + docosahexaenoic acid; GI, gastrointestinal; HTN, hypertension; $\mathrm{KCl}$, potassium chloride; NA, not available; NR, not reported; PAD, peripheral artery disease; RCT, randomized controlled trial; SBP, systolic blood pressure; SR/MA, systematic review and meta-analysis.

of flax seed with placebo in patients with and without hypertension and found marked decreases in systolic and diastolic BP. ${ }^{7}$ Study participants were all older than 40 years without other major cardiac or renal disease, and the majority of enrolled patients with hypertension were concurrently taking medications to treat hypertension during the study.

I Olive leaf extract. A double-blind, parallel, and active-control clinical trial in Indonesia compared the BP-lowering effect of olive leaf extract (Olea europaea) to captopril as monotherapies in patients with stage 1 hypertension. ${ }^{8}$ After a 4 -week period of dietary intervention, individuals who were still hypertensive (range, 140/90 to $159 / 99 \mathrm{~mm} \mathrm{Hg}$ ) were treated with either olive leaf extract or captopril. After 8 weeks of treatment, both groups saw comparable reductions in BP.

\section{Editor's takeaway}

Many studies have demonstrated BP benefits from a variety of natural supplements. Although the studies' durations are short, the effects sometimes modest, and the outcomes disease-oriented rather than patientoriented, the findings can provide a useful complement to our efforts to manage this most common chronic disease.

JFP

\section{References}

1. Ried K, Fakler P, Stocks NP. Effect of cocoa on blood pressure. Cochrane Database Syst Rev. 2017;(4):CD008893.

2. Miller PE, Van Elswyk M, Alexander DD. Long-chain omega-3 fatty acids eicosapentaenoic acid and docosahexaenoic acid and blood pressure: a meta-analysis of randomized controlled trials. Am J Hypertens. 2014;27:885-896.

3. Rohner A, Ried K, Sobenin IA, et al. A systematic review and meta-analysis on the effects of garlic preparations on blood pressure in individuals with hypertension. Am J Hypertens. 2015;28: 414-423.

4. Poorolajal J, Zeraati F, Soltanian AR, et al. Oral potassium supplementation for management of essential hypertension: a meta-analysis of randomized controlled trials. PLOS One. 2017;12:e174967.

5. Dong JY, Qin LQ, Zhang Z, et al. Effect of oral L-arginine supplementation on blood pressure: a meta-analysis of randomized, double-blind, placebo-controlled trials. Am Heart J. 2011;162:959-965.

6. Kapil V, Khambata RS, Robertson A, et al. Dietary nitrate provides sustained blood pressure lowering in hypertensive patients: a randomized, phase 2, double-blind, placebo-controlled study. Hypertension. 2015;65:320-327.

7. Rodriguez-Leyva D, Weighell W, Edel AL, et al. Potent antihypertensive action of dietary flaxseed in hypertensive patients. Hypertension. 2013;62:1081-1089.

8. Susalit E, Agus N, Effendi I, et al. Olive (Olea europaea) leaf extract effective in patients with stage-1 hypertension: comparison with captopril. Phytomedicine. 2011;18:251-258. 